\title{
Novel Loudspeaker and Headphone Design Approaches Enabled by Adaptive Nonlinear Control
}

\author{
WOLFGANG KLIPPEL, ${ }^{1}$ AES Fellow \\ (w.klippel@klippel.de) \\ ${ }^{1}$ Klippel GmbH, Dresden, Germany
}

\begin{abstract}
Traditional loudspeaker and headphone design sacrifices efficiency for sound quality. Nonlinear, adaptive control can compensate for undesired signal distortions, protect the transducer against overload, stabilize the voice coil position, and cope with time-varying properties of the suspension. This paper discusses the consequences of new software opportunities for designing optimum hardware components that exploit available resources, such as a nonlinear voice coil gap configuration, a softer suspension, and modal vibrations in the diaphragm, panel, and in the acoustical systems to increase the efficiency of the electroacoustical conversion. Digital signal processing (DSP) software complementing transducer hardware is the key to modern audio devices generating the required sound output with minimum size, weight, cost, and energy.
\end{abstract}

\section{INTRODUCTION}

The progress of digital signal processing (DSP) provides new opportunities for manipulating the input signal supplied to the transducer used in loudspeakers and headphones to generate more sound output, a desired directivity and less distortion while actively protecting the transducer against thermal and mechanical overload. Software can provide solutions with self-learning capabilities that monitor the properties of the transducer, generate the optimal control output, and adaptively react to changing conditions. Furthermore, DSP becomes, with higher integration, more affordable, and virtual changes realized by software modifications simplify the product optimization and speed up the development process.

On the other side, DSP cannot directly improve the electroacoustical efficiency of the transducer, which is a critical point in sound reproduction. Most of the electrical input power will be converted into heat, and only a small fraction (far below $1 \%$ in microspeakers) will be converted into sound power. The Green Speaker Design [1] uses available DSP functionality with a modified hardware to generate more benefits for the end user while using less resources such as material (e.g., magnet), energy, and manufacturing effort. Generating the desired sound output with higher efficiency while reducing size, weight and cost are the key criteria in Green Speaker Design [2].
This paper gives an overview on the new software opportunities and discusses further consequences for the hardware design and shows the benefit of using active transducer modules in future audio products. The focus is set on conceptional frameworks, benefits, practical concerns, possible trends, and the relationship to other audio technologies. The interested reader will be referred to primary research papers which describe the theory, implementation, and practical evaluation in greater detail.

\section{NEW SOFTWARE OPPORTUNITIES}

An electroacoustical transducer is a highly nonlinear and time-variant system. Adaptive, nonlinear control is required to compensate for the undesired properties and to generate a desired transfer behavior.

Fig. 1 shows a block diagram of such a control system applied via an amplifier with current sensing to a transducer operated in a loudspeaker, headphone, or any other active audio device. The control system generates a desired linear transfer function between digital audio input $w(t)$ and sound pressure output $p(t, \mathbf{r})$, adjusted to variations over product life. It comprises four subsystems operated at full sample rate (e.g., $48 \mathrm{kHz}$ ) and with sufficient resolution ( $\geq 24$ bits).

The first subsystem is a linear filter for equalizing the frequency response. The second is a time-variant, linear system providing active protection of the transducer against 


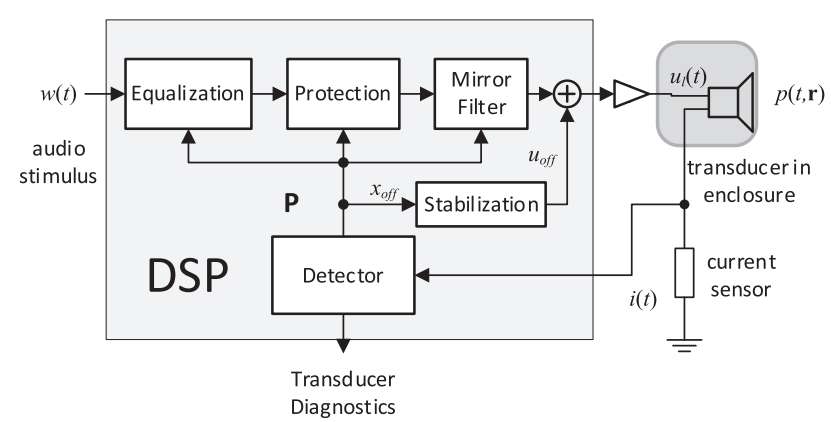

Fig. 1. Block diagram of an active loudspeaker system with adaptive, nonlinear control of the transducer based on Klippel Controlled Sound (KCS) technology [27].

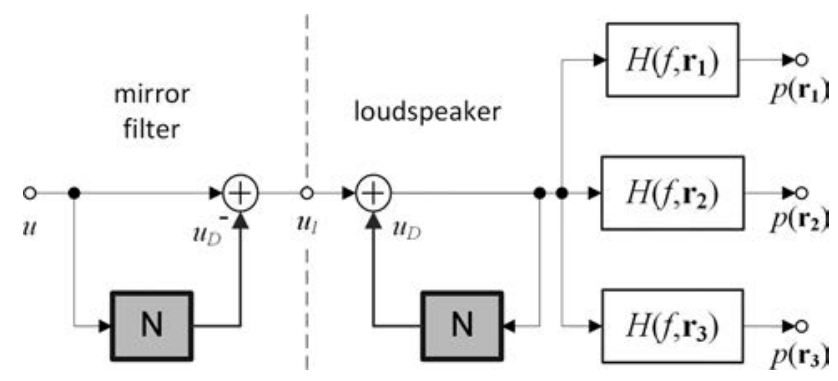

Fig. 2. Active linearization of the passive audio system by using the mirror filter [4].

mechanical and thermal overload. The following mirror filter linearizes the loudspeaker by canceling the nonlinear distortion in the output signal. The last subsystem stabilizes the voice coil rest position in order to generate maximum voice coil displacement. The power amplifier has a constant gain and is preferably DC-coupled to avoid attenuating lowfrequency compensation signals generated by the nonlinear control.

The transducer input current $i(t)$ measured by an electrical sensor (e.g., resistor) is used for identifying the timevariant properties of the transducer and adjusting the audio processing to the varying conditions. Thus, the nonlinear adaptive control system uses a feedback of updated transducer parameters $\mathbf{P}$. The detector can perform the parameter identification at a reduced sample rate $(6-12 \mathrm{kHz})$ and is immune to any latency in the sensing path. The parameters $\mathbf{P}$ have a physical meaning, a high diagnostic value, and can be stored in a memory.

\subsection{Active Distortion Cancellation}

There are different solutions for linearizing transducers by processing the digital input signal as described in [3]. The mirror filter [4] is a very practical approach because it uses an accurate control law derived by electroacoustical modeling, predicts all required state variables based on the audio input signal $u(t)$, and generates low processing load.

The signal flow chart in Fig. 2 shows a subsystem N that generates nonlinear distortion $u_{D}(t)$ added to the loudspeaker input signal $u_{l}(t)$ forming a feedback loop. This model describes the nonlinear dynamics of the force factor

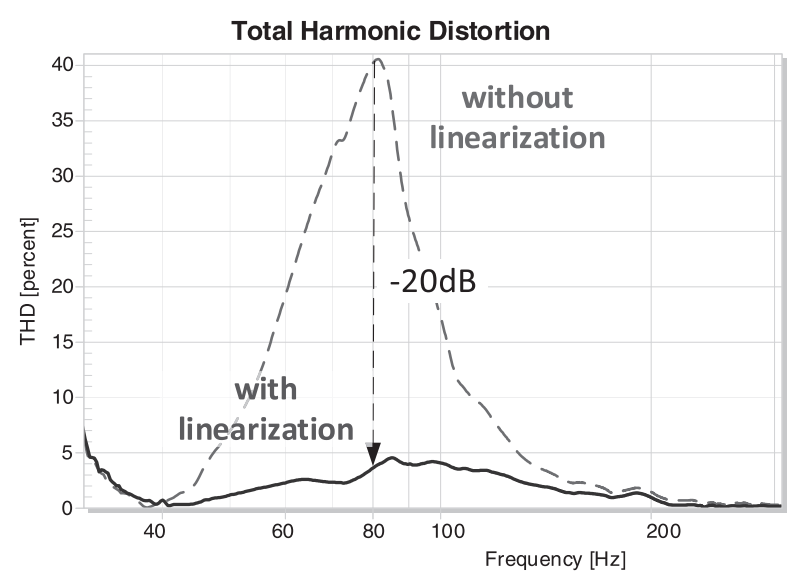

Fig. 3. Total harmonic distortion in the sound pressure output of an automotive speaker (transducer B in [2]) measured without and with linearization using the mirror filter.

$B l(x)$, suspension stiffness $K_{M S}(x)$, mechanical resistance $R_{M S}(v)$, inductance $L(x, i)$, and other speaker nonlinearities, which are lumped parameters depending on voice coil displacement $x$, velocity $v$, input current $i$, and other state variables.

The mirror filter uses a copy of the subsystem $N$ connected to the filter input $u(t)$ in a feed-forward arrangement to synthesize a compensation signal $-u_{D}(t)$ that cancels the equivalent input distortion $u_{D}(\mathrm{t})$ generated in the loudspeaker. The result is a linear transfer function $H(f, \mathbf{r})$ between filter input $u(t)$ and sound pressure output $p(t, \mathbf{r})$. All harmonic and intermodulation distortions are cancelled at any observation point $\mathbf{r}$ [5], and the DC displacement and instabilities of nonlinear dynamics are removed. The distortion compensation not only improves the perceptual sound quality but also the performance of acoustic echo cancellation, active noise attenuation, and other digital algorithms that require linear electroacoustical transduction [6].

In practice, the transducer model is restricted to the dominant nonlinearities found in the particular audio product to reduce the processing load and the cost of the product. Due to those practical limitations the distortion reduction is typically $6-20 \mathrm{~dB}$ for dominant distortion.

Fig. 3 shows, for example, a $20-\mathrm{dB}$ reduction of the total harmonic distortion at $80 \mathrm{~Hz}$ generated by a loudspeaker designed for pedestrian alert systems in electric cars [2]. At this frequency, the sinusoidal stimulus generates maximum voice coil displacement $x$ and activates the dominant nonlinearities force factor $B l(x)$, stiffness $K_{\mathrm{ms}}(x)$, and inductance $L(x)$. At lower frequencies a fourth-order alignment realized by active equalization reduces the voice displacement $x$, and the distortion becomes negligible. The displacement $x$ also decreases at higher frequencies, and the dominant nonlinearities generate less harmonic distortion. This is not the case for a multitone stimulus representing a typical audio signal, in which low-frequency signals modulate higher-frequency signals. Those broadband intermodulation distortions can also be significantly reduced 


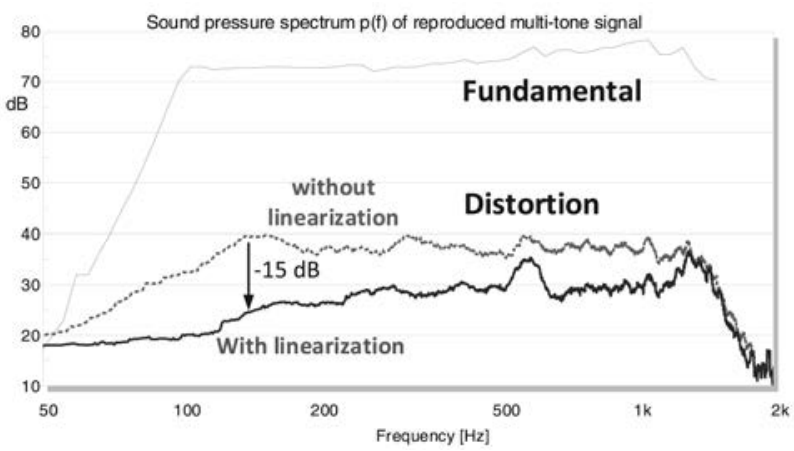

Fig. 4. Fundamental components and nonlinear distortion in a multitone test stimulus reproduced by an automotive speaker (transducer B in [2]) without and with linearization using the mirror filter.

by the mirror filter as shown for the same loudspeaker in Fig. 4.

The active linearization of the loudspeaker should be applied to a defined excursion range $|x(t)|<x_{\text {mech }}$ limited by the mechanical limit $x_{\text {mech }}$ to avoid impulsive distortion generated by transducer irregularities (rub and buzz). Most of the impulsive distortion is not deterministic and cannot be cancelled by nonlinear control.

The feed-forward structure of the mirror filter is always stable and generates no latency in the audio signal path. It comprises a minimum of elements (dynamic linear elements and static nonlinearities) that correspond to the states and parameters of the electroacoustical model [5].

\subsection{Stabilization of Coil Rest Position}

The rest position of the voice coil depends on the mechanical suspension system, which depends on the manufacturing process, influence of climate and gravity, fatigue, and aging. A small offset can generate significant DC displacement, high distortion, and other symptoms of instability.

The stabilization block in Fig. 1 synthesizes a DC compensation voltage $u_{o f f}(t)$ added to the output of the mirror filter to compensate for the offset $x_{o f f}(t)$ in the voice coil rest position, giving maximum $\mathrm{AC}$ displacement and sound output over its lifetime.

The absolute position of the voice coil of a Device Under Control (DUC) can be described as

$$
X_{a}(t)=X_{0}+x_{o f f}(t)+x_{d i s}(t)+x_{l i n}(t)
$$

with the reference rest position $X_{0}$ of the approved prototype or Golden Reference Device (GRD), an offset $x_{o f f}(t)$ in the rest position of the particular unit due to production variances and unknown influences (e.g., gravity), nonlinear distortion $x_{d i s}(t)$ generated dynamically by the transducer, and the linear displacement $x_{\text {lin }}(t)$.

The displacement of the voice coil from the rest position

$$
\begin{gathered}
x(t)=X_{a}(t)-X_{0} \\
=x_{o f f}(t)+x_{D C}(t)+x_{A C}(t)
\end{gathered}
$$

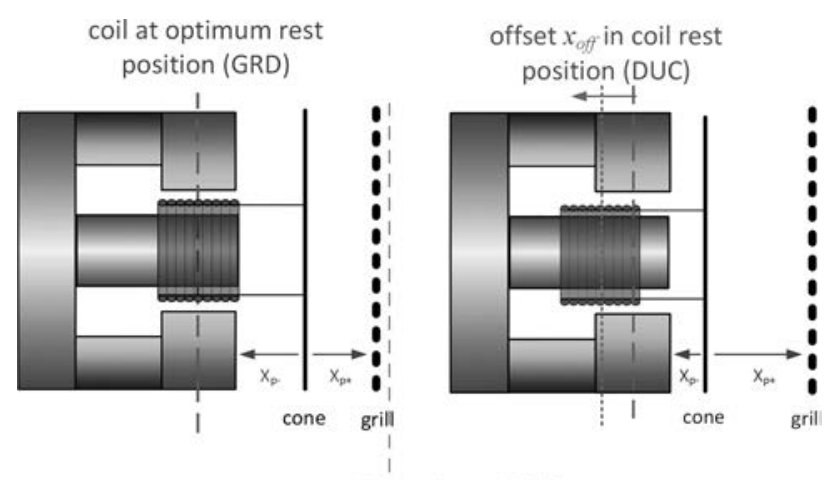

Force factor $\mathrm{BI}(\mathrm{X})$

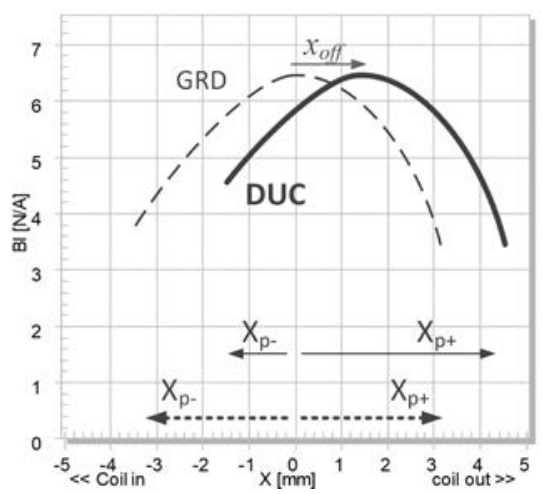

Fig. 5. An offset in the coil rest position of a particular Device Under Control (DUC) to the Golden Reference Device (GRD) causes a shift in the force factor curve $B l(x)$ versus displacement $x$

comprises time-varying components, of which only the AC component $x_{A C}(\mathrm{t})$ generates sound. The DC displacement $x_{D C}(t)$ is a low-frequency component dynamically generated by the rectification of the audio stimulus in the transducer nonlinearities [5]. It can be cancelled when the mirror filter output is transferred to the transducer via a DCcoupled amplifier.

Both the offset $x_{o f f}(t)$ and the DC displacement $x_{D C}(t)$ decrease the sound pressure output because either the positive or the negative peak displacement $X_{p+}$ and $X_{p-}$ is reduced. The offset $x_{o f f}(t)$ in the voice coil rest position of a particular DUC can be detected as a shift in the force factor characteristic $B l(x)$ of the DUC and GRD as shown in Fig. 5. This relation is described in more detail in [7].

\subsection{Transducer Overload Protection}

Furthermore, modern amplifiers can provide more power than the transducer can usually handle. To avoid mechanical and thermal overload, active protection systems [8], [9] have been developed that detect a critical state and attenuate the input signal before damage occurs. The protection system in Fig. 1 uses the displacement $x$ and temperature $T_{v}$ of the coil, which is reliably predicted in the mirror filter, in combination with thermal modeling [10] and adaptive parameter measurement [11].

The protection system behaves like a linear time-variant filter that provides a flat response with $H_{\text {prot }}(f)=1$ if no mechanical or thermal overload is anticipated. To keep the 
voice coil displacement $x$ in a permissible range $|x|<x_{\text {prot }}$, the filter temporarily generates a high-pass characteristic in $H_{\text {prot }}(f)$ attenuating low frequency audio components, which are less audible but contribute significantly to the total displacement $x$. Predictive modeling of the peak displacement $x_{\text {peak }}=\max (x(\mathrm{t}))$ can be used to activate the attenuation filter in advance and to reduce audible artifacts during the attack phase [8].

Thermal protection requires an attenuation of the total input signal or signal components that significantly contribute to the real input power $P_{e}$.

\subsection{Self-Learning of Initial Parameters}

The digital audio processing discussed so far has a feedforward structure and is immediately operative after powering up the system by using an initial control setup comprising the parameters $\mathbf{P}_{\text {init }}$ measured once on the GRD of that device type [14]. The GRD can be the final prototype at the end of the hardware development or found by performing statistical analysis of the data collected during end-of-line testing in transducer manufacturing. The initial identification process automatically determines the usable working range by measuring the maximum peak displacement $\mathrm{X}_{\mathrm{MAX}}$ and evaluating the impulsive distortion (rub and buzz).

\subsection{Adaptive Parameter Adjustment}

Although the control system can be operated with the initial parameters, any mismatch between the control parameters and the actual loudspeaker parameters will deteriorate the performance. Thus, an adaptive parameter adjustment [14] in the detector in Fig. 1 is required to cope with unavoidable production variances, aging of the suspension [15], changing climate conditions, and other external influences.

All lumped parameters of the nonlinear loudspeaker model can be identified by an inexpensive electric sensor monitoring the input current $i(t)$ as described in [11]. The adaptive control system also simplifies the tuning process in active systems and provides accurate transducer parameters over the lifetime of the product while reproducing music or other audio signals.

\subsection{Equalization of the Overall Response}

Traditional alignment in the passive system design [12] searches for a particular transducer and the optimum shape of the cabinet to generate a desired shape of the transfer response $H_{o}(f, \mathbf{r})$ defined as a generalized high-pass system function (e.g., Butterworth).

Equalization [13] gives more freedom in the hardware design because a linear filter with the transfer function $H_{\text {equ }}(f)$ cancels undesired poles and zeros in the rational transfer function $H(f, \mathbf{r})$ of the passive system and introduces new ones to generate any desired overall system function:

$$
H_{o}(f, \mathbf{r})=H_{e q u}(f) H(f, \mathbf{r})
$$

Adaptive, nonlinear control automatically calculates the optimum filter function $H_{\text {equ }}(f)$ based on the electroacoustical model and the initial parameters measured on the GRD
[1]. This alignment is valid for all DUCs operated at small and high amplitudes because the mirror filter also compensates for linear variations in stiffness, damping and other lumped parameters caused by production and time variances [4].

\section{CONSEQUENCES FOR HARDWARE DESIGN}

The new software opportunities give new degrees of freedom for the optimization of the hardware components which sets new priorities in the transducer and audio system design.

\subsection{Size, Shape, and Weight}

Most end-users prefer small and light audio devices for personal and portable applications. The size and sometimes even the shape of the hardware components affecting the sound radiation and propagation are already defined by marketing, artistic design, and manufacturing before the electroacoustical development begins.

\subsection{Electroacoustical Efficiency}

In general, the electroacoustical efficiency is defined as the ratio

$$
\eta=\frac{P_{a}}{P_{e}} 100 \%
$$

between acoustic output power $P_{a}$ and electric input power $P_{e}$. This characteristic is a key criterion in Green Speaker Design [1] to optimize the design with respect to energy consumption, maximum output and heat development. Efficiency depends on the particular stimulus and applied signal processing and becomes essential in modern audio devices using significant equalization (e.g., bass boost) to extend the usable frequency range.

By representing the statistical properties of audio stimulus as the spectrum $G_{w}(f)$ and assuming a bell-shaped amplitude distribution of the transducer state variables [1], the efficiency $\eta$ in Eq. (4) can also be expressed as

$$
\eta=\frac{\int_{0}^{+\infty} \eta(f) \Re\left(Z_{E}(f)^{-1}\right) G_{w}^{2}(f) d f}{\int_{0}^{+\infty} \Re\left(Z_{E}(f)^{-1}\right) G_{w}^{2}(f) d f}
$$

with a frequency dependent efficiency function

$$
\eta(f)=\frac{\left|H\left(f, r_{r e f}\right)\right|^{2} 4 \pi r_{r e f}^{2}}{\rho_{0} c \Re\left(Z_{E}(f)^{-1}\right) Q\left(f, r_{r e f}\right)} 100 \%
$$

using the specific acoustic impedance $\rho_{0} c$. The complex transfer function $H\left(f, \mathrm{r}_{r e f}\right)$ between a sinusoidal generator voltage $u_{\mathrm{g}}$ and sound pressure $p\left(t, \mathrm{r}_{e f}\right)$ at a distance $r_{r e f}$ on-axis from the source and the electrical impedance $Z_{E}(f)$ increases the efficiency. A higher directivity factor $Q\left(f, r_{r e f}\right)$ reduces the efficiency.

\subsubsection{Lumped Parameter Optimization}

The sound pressure response $H\left(f, \mathbf{r}_{r e f}\right)$ and electrical impedance $Z_{E}(f)$ can be calculated by lumped parameter 


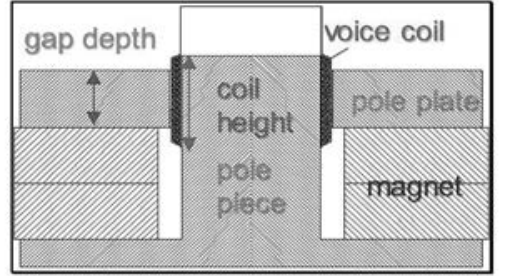

optimum-length configuration

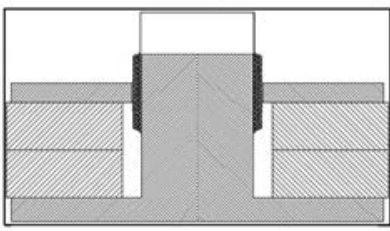

over-hung coil

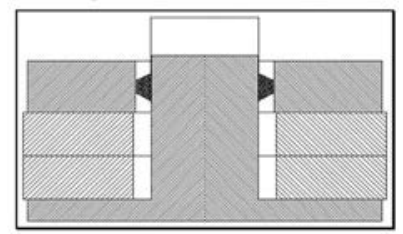

under-hung coil
Fig. 6. Common topologies for configuring the gap depth and voice coil height in electrodynamical transducers.

modeling as shown in [1] for the electrodynamical transducer used in sealed as well as various vented enclosures with and without passive radiators. This model is valid at low and medium frequencies because the fundamental (piston) mode dominates the mechanical vibration and the acoustical wavelength is larger than the geometrical dimension of the audio device, generating an almost omnidirectional directivity pattern $(Q \asymp 1)$.

Since adaptive, nonlinear control always generates the desired target overall response $H_{o}(f, \mathbf{r})$ for any choice of the transducer and enclosure parameters, this freedom can be used to increase the efficiency by the following ways:

A lower stiffness $K_{M S}$ of the mechanical suspension reduces the fundamental resonance frequency $f_{0}$ and increases $\eta(f)$ for $f<f_{0}$.

A larger effective radiation area $S_{D}$ increases the efficiency $\eta(f)$ in many applications (e.g., vented box) but not in a subwoofer operated at low frequencies $f<f_{0}$ in a small sealed box, where the equivalent stiffness $K_{M B}$ of the enclosed air is larger than the stiffness $K_{M S}$ of the suspension. In this case, a larger peak displacement $X_{M A X}$ of a smaller diaphragm can generate the required displaced air volume $S_{D} X_{M A X}$ more efficiently [1].

A lower value of the moving mass $M_{M S}$ increases both the transfer function $H\left(f, \mathrm{r}_{r e f}\right)$ and the efficiency $\eta(f)$ above the fundamental resonance frequency $f_{0}$ as discussed in [2].

Although a larger volume $V_{A B}$ of the enclosed air increases the efficiency of the transducer mounted in sealed or vented enclosures with and without passive radiator, the geometrical design of the acoustical system is limited by other nonacoustical constraints to satisfy the expectations of the customer.

The active distortion cancellation sets the transducer nonlinearities in a new light.

Fig. 6 shows typical coil-gap configurations that give either more efficiency or improved linearity of the electrodynamical motor. The optimum-length configuration uses a small voice coil overhang to exploit the induction inside the gap and the major part of the fringe field outside the

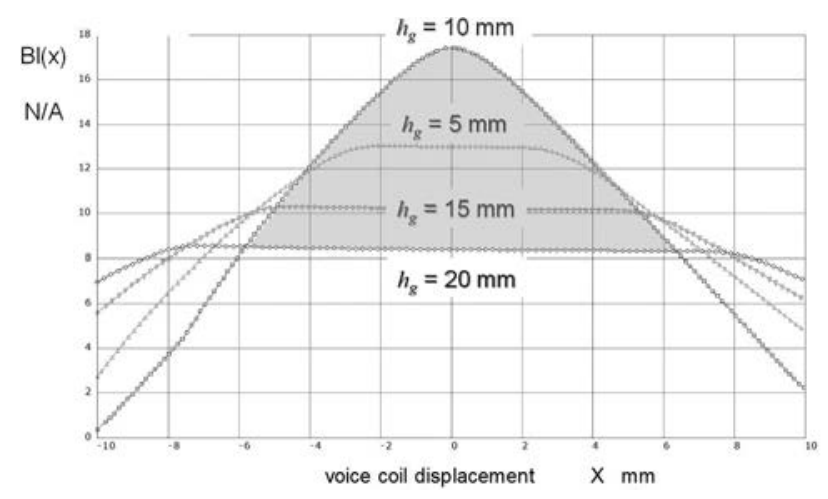

Fig. 7. Force factor $B l(x)$ versus displacement $x$ of an equal-length, an overhung, and an underhung configuration using a constant voice coil height $h_{c}=10 \mathrm{~mm}$ and different gap depths $h_{g}$.

gap. A small displacement of the coil from the optimum rest position will reduce the mean induction seen by coil. The overhung and underhung configurations keep, at moderate coil displacement, an almost constant number of coil windings in the gap.

Fig. 7 shows the nonlinear force factor characteristic $B l(x)$ calculated by a magnetic finite element modeling where the gap depth $h_{g}$ was varied between 5,10,15 and $20 \mathrm{~mm}$ while using the same magnet and a constant coil height $h_{c}=10 \mathrm{~mm}$ [2].

The equal-length configuration $h_{c}=h_{g}$, which is close to the optimum-length configuration, gives the largest force factor at the rest position $x=0$ in Fig. 7 but generates an early decay of the force factor $B l(x)$ if the coil is moved away from the optimal rest position. This nonlinearity causes significant harmonic and intermodulation distortion in the output signal and motor instabilities generating a high DC displacement [5].

The additional windings below and above the gap in the overhung coil configuration $\left(h_{g}=5 \mathrm{~mm}\right)$ generate a plateau in the $B l(x)$ characteristic in Fig. 7, giving more linearity and less nonlinear distortion for moderate excursion. A similar result can be achieved by using a 15 or 20 -mm-thick pole plate in the underhung coil configurations.

The price paid for less $B l(x)$ variation and lower nonlinear distortion is a loss of force factor, illustrated as a shaded area in Fig. 7.

The influence of the electrodynamical motor on the efficiency in Eq. (5) can be described by the motor efficiency factor defined as

$$
\frac{(B l)^{2}}{R_{E}}=\bar{B}^{2} \frac{V_{w}}{\kappa_{w}}
$$

which is the ratio between squared force factor $B l^{2}$ and DC resistance $R_{E}$. Alternatively, it is calculated in [2] based on the electrical resistivity $\kappa_{w}$, the volume $V_{w}$ occupied by the conductive wire material, and the mean induction $\bar{B}$, which represents the induction $B$ averaged over wire length $l$.

The mean induction $\bar{B}$ depends on the relationship between the gap depth $h_{g}$ and the voice coil height $h_{c}$. The optimum-length configuration gives the highest mean induction $\bar{B}$, which is close to the maximum induction value 
$B_{\text {max }}$ found in the gap. For a given magnet volume $V_{m}$, the motor efficiency factor can be calculated as follows:

$$
\frac{(B l)^{2}}{R_{E}}=\frac{\mu_{0} B_{m} H_{m} \sigma V_{m}}{\kappa_{w}} \frac{V_{w}}{V_{g}}
$$

Modern magnetic Finite Element Analysis (FEA) design tools can be used to maximize this factor in the following way:

The gap width is optimally adjusted to the height of the magnet, where the field strength $H_{m}$ and induction $B_{m}$ in the magnet comes close to the maximum energy product $(B H)_{\max }$, which is an important material parameter of the magnet material [2]. It is also important to maximize the stray field factor $0<\sigma<1$ that represents the ratio between the flux passing the pole tips and total flux generated by the magnet.

The ratio $V_{w} / V_{g}$ between wire volume and effective gap volume in Eq. (8) can be interpreted as a filling factor, where $V_{w} / V_{g}<1$ due to the bobbin and the clearance between the coil and the pole tips is required to cope with rocking modes and voice coil rubbing. The fill factor can be increased by using rectangular wire and an efficient winding layout [18], which usually increases the manufacturing effort and cost.

Maximizing the motor efficiency factor $(B l)^{2} / R_{E}$ is an important target in Green Speaker Design as illustrated on an automotive speaker in [2]. A reduced voice coil overhang improved the pass band efficiency by $65 \%$ and the maximum sound pressure level SPL MAX by $3 \mathrm{~dB}$ while the adaptive, nonlinear control reduced the signal distortion by more than $15 \mathrm{~dB}$.

\subsubsection{Exploiting Modal Vibrations}

Modal resonances in the mechanical system generate poles in the transfer function $H\left(f, r_{r e f}\right)$ in Eq. (6), which improve the efficiency of the audio system at the natural frequencies $f_{n}$ as discussed for a panel speaker in [1]. The sound radiation generates additional zeros in a complex mechanoacoustical transfer function $S_{D}(f)$ which can be interpreted as an effective radiation area describing the relationship between coil velocity $v$ and the generated volume velocity $q_{a}$. The poles and zeros in $S_{D}(f)$ can be perfectly equalized by linear filtering giving a flat volume velocity response. Unfortunately, the pole setting varies over manufactured units and over time. The active equalization would benefit from an adaptive adjustment based on sound pressure measurement [1].

Furthermore, the sound pressure transfer function $H(f, \mathbf{r})$ varies at higher frequencies with the position of the observation point $\mathbf{r}$. In practice, the equalization of higherorder modes requires a compromise in the flatness of the sound pressure response averaged in a defined listening zone. Modern finite element simulation tools combined with laser vibrometry and modal analysis can be used to improve the efficiency at higher frequencies while generating a sufficiently flat response and a desired directivity factor $Q(f)$.

\subsection{Maximum Sound Output}

The optimization of the transducer efficiency is not only important for reducing the energy consumption and extending the operation time of battery-powered audio devices but it is also the key for increasing the maximum acoustical output for a given power amplifier. The following approaches optimize the maximum value of the Sound Pressure Level (SPL) and acoustical power under transient and steady-state conditions:

\subsubsection{Improved Heat Flow}

The thermal overload protection system attenuates the audio signal to prevent that the voice coil temperature $T_{v}$ exceeds a predefined limit $T_{l i \mathrm{~m}}$. The transducer design can reduce the coil temperature not only by reducing the energy consumption but also by exploiting forced air convection and other cooling mechanisms [9]. The thermal capacity corresponding to the mass of the coil, iron path, magnet, and frame determines the time constant of the thermal dynamics, which is important to cope with peaks in the input power.

\subsubsection{Transducer-Amplifier Matching}

In practice, either the maximum peak voltage $u_{\max }$ or the maximum peak current $i_{\max }$ at the amplifier output limits the maximum sound pressure peak value [17]. The best compromise can be found by matching the expectation value $\overline{Z_{E}}$ of the electrical impedance $Z_{E}(f)$ with the ratio of the maximum peak values of the amplifier output:

$$
\frac{u_{\max }}{i_{\max }} \approx \overline{Z_{E}}=\frac{\sqrt{\int_{0}^{+\infty} G_{u}^{2}(f) d f}}{\sqrt{\int_{0}^{+\infty} G_{u}^{2}(f)\left|Z_{E}(f)\right|^{-2} d f}}>R_{E}
$$

The expectation value $\overline{Z_{E}}$ is similar to the nominal impedance $Z_{n}$ as defined in common standards [19] which is typically 4 or $8 \mathrm{Ohm}$ and does not consider the typical voltage spectrum $G_{u}(f)=\left|H_{\text {equ }}(f)\right| G_{w}(f)$ at the speaker terminals as a weight of $Z_{E}(f)$.

An electrodynamical transducer with optimum-length configuration as shown in Fig. 6 can be easily matched to the amplifier by selecting a wire diameter that gives DC resistance $R_{E}$ required to generate the impedance value $\overline{Z_{E}}$ needed by the power amplifier. This modification of the DC resistance will not change the motor efficiency factor $B l^{2} / R_{E}$ if the wire volume $V_{w}$ stays the same [2].

\subsubsection{More Voltage Sensitivity}

In subwoofers operated in a narrow band around the fundamental resonance frequency $f_{0}$, the mean impedance $\overline{Z_{E}}$ can easily exceed $20 \mathrm{Ohm}$ and generates high peak voltage requirements for the amplifier to feed the required power into the transducer. Considering the influence of the 
stimulus, the voltage sensitivity can be defined as the total sound pressure level in $\mathrm{dB}$

$$
\begin{gathered}
L_{u_{r e f}, r_{r e f}}=20 \lg \left(\frac{p_{r m s}\left(r_{r e f}\right)}{p_{0}}\right) \\
=10 \lg \left(\frac{1}{p_{0}^{2}} \int_{0}^{+\infty} G_{u}^{2}(f)\left|H\left(f, r_{r e f}\right)\right|^{2} d f\right)
\end{gathered}
$$

at a given reference distance on-axis (typically $r_{\text {ref }}=1$ $\mathrm{m})$ with the reference sound pressure $p_{0}=2 \cdot 10^{-5} \mathrm{~Pa}$ considering the amplitude spectrum $G_{u}(f)$ corresponding to a reference RMS value of the generator voltage (typically $\left.u_{\text {ref }}=1 \mathrm{~V}\right)$ :

$$
u_{r e f}^{2}=\int_{0}^{+\infty} G_{u}^{2}(f) d f
$$

For a sinusoidal stimulus at frequency $f$, a frequencydependent voltage sensitivity function can be expressed as

$$
L_{u_{r e f}, r_{r e f}}(f)=20 \lg \left(\left|H\left(f, r_{r e f}\right)\right| \frac{u_{r e f}}{p_{0}}\right)
$$

which is a useful characteristic for transducer design [12].

Comparing Eqs. (5) and (10) shows that efficiency and voltage sensitivity are related but not identical [1]. Both characteristics contain the on-axis transfer response $H\left(f, r_{r e f}\right)$ that generates a similar curve shape at low and medium frequencies. However, the voltage sensitivity can be increased by reducing the DC resistance $R_{E}$. Practically speaking, reducing the DC resistance to half of its value gives $3 \mathrm{~dB}$ more voltage sensitivity but also increases the input current by $3 \mathrm{~dB}$ [2].

The general definition of the voltage sensitivity in Eq. (10) allows the maximum sound pressure level value $S P L_{\max }$ to be calculated considering the properties of the stimulus.

\subsubsection{Maximum Displacement}

The mechanical overload protection system in the DSP attenuates the low frequency components to prevent an overshoot of the voice coil displacement over a defined limit $X_{M A X}$. The displaced air volume $S_{D} X_{M A X}$ and the acoustical system type (e.g., vented box) limits the maximum SPL at low frequencies. A vented enclosure or a passive radiator can reduce the displacement of the transducer at the acoustical resonance.

The voice coil height $h_{c}$ limits the maximum displacement $X_{M A X}$ in electrodynamical transducers. According to International Electrotechnical Standard (IEC) standard 62458 [20], the force factor $B l\left(X_{M A X}\right)$ referred to the force factor value at the rest position $B l(x=0)$ drops at a peak displacement $X_{M A X}$ to a force factor ratio $B l_{\min }$ defined as

$$
B l_{\min }=\min _{-X_{M A X}<x<X_{M A X}}\left(\frac{B l(x)}{B l(0)}\right) \cdot 100 \%
$$

For example, moving the coil to a force factor ratio $B l_{\text {min }}=82 \%$ generates about $10 \%$ inter-modulation distortion for a two-tone signal [20].
If nonlinear distortion cancellation can be applied to the transducer, the minimum force factor ratio can be reduced to $B l_{\text {min }}=50 \%$, giving a larger peak displacement $X_{M A X} \asymp$ $h_{c} / 2$. At this point, half of the coil windings are out of the gap if there is no offset in the coil's rest position.

The minimum ratio of the mechanical compliance $C_{M S}(x)$ referred to the compliance at the rest position $C_{M S}(0)$ defined as

$$
C_{\min }=\min _{-X_{M A X}<x<X_{M A X}}\left(\frac{C_{M S}(x)}{C_{M S}(0)}\right) \cdot 100 \%
$$

should be larger than $50 \%$ to generate a small distortion cancellation signal $u_{D}$ in the mirror filter and to avoid a significant increase of the crest factor in the terminal voltage $u_{l}$.

The transducer should generate the maximum displacement $X_{M A X}$ without generating significant impulsive distortion measured according IEC standard [21] which indicates hard limiting of the suspension, voice coil rubbing, and other irregular transducer behavior.

\subsection{Desired Directivity}

The DSP applied to the input signal of a single transducer cannot change the directivity and other 3D characteristics of the generated sound field. A proper design of the cone, diaphragm, panel and other hardware components using FEA, Boundary Element Analysis (BEA), modal analysis, and other numerical methods is needed to optimize the mechanical vibrations for a desired performance [1]. Unfortunately, the size of the radiating surface limits the modification of the directivity pattern.

Loudspeaker arrays comprising multiple transducers with separate DSP processing [22] provide more flexibility for changing the directivity pattern and actively forming and steering one or more sound beams with different audio content to a particular listening area. Good performance, especially at high frequencies, requires small transducers that are closely spaced to fulfill the sampling criteria at short wavelength [22]. Loudspeaker arrays also need efficient transducers to avoid heat problems at high amplitudes because the air load resistance seen by each transducer becomes smaller when operated with other transducers together in an array. The active cancellation of the nonlinear distortion improves the acoustical contrast between dark and bright sound zones as discussed in [23]. The adaptive control copes with different transducer properties due to production variances and ensures optimum performance over time. The signal processing (delay, attenuation, or filtering) required for beam forming can be combined with speaker alignment, crossover filter, and other equalization in the first linear block of the adaptive nonlinear control.

\subsection{Simplified Manufacturing}

There are close links and synergistic effects between adaptive, nonlinear control and manufacturing.

The measurement results collected during end-of-line testing of the transducers and assembled audio devices are a convenient basis for selecting a golden reference device 
(GRD) and generating an optimal initial control setup [24]. If the parameter deviation between the devices under test (DUTs) in the End-of-Line (EoL)-test is sufficiently small, the same initial setup can be applied for all devices under control (DUCs).

The spider and surround are critical soft parts in transducer manufacturing that generate significant deviations of the stiffness $K_{M S}$, resonance frequency $f_{s}(\mathrm{DUT})$, total quality factor $Q_{T S}$ (DUT), and an offset $x_{o f f}$ (DUT) in the voice coil rest position of the particular DUT from the mean value. Those production variances are still smaller than the parameter changes occurring during break-in and over the further product life time. Since the adaptive nonlinear control can compensate those deviations of the DUC, larger tolerances can be applied in the EoL-testing of DUTs, which increase the yield rate and reduce the cost of transducer manufacturing. However, a transducer (DUT) with extreme deviation in offset $x_{\text {off }}$ and resonance frequency $f_{s}$ should be rejected because the DUC has a significantly reduced efficiency.

The adaptive, nonlinear control activated in the particular DUC for the first time provides unique diagnostic information from the electromechanical system which complement the results of the acoustical testing. A comprehensive selftest of the DUC can be realized by using the internal microphone, amplifier with current sensing, and DSP available in some audio devices (e.g., smartphones and smart speakers).

The acoustical test checks the performance of the audio device at higher frequencies which cannot be detected by current sensing. The most critical test is the standard measurement for impulsive distortion [21] to reliably detect buzzing transducer parts, loose particles, coil rubbing, open glue joint, air leakages, and other abnormal behavior. Transducers and assembled audio devices showing those symptoms cannot be shipped to the customer because those defects usually become worse over time [24]. Analyzing the dependency of the impulsive distortion on the voice coil position and other transducer properties provided by the detector gives a deeper understanding and clues for fixing the root cause of the impulsive distortion.

\subsection{Maximum Endurance and Reliability}

Steps must be taken to avoid failures in the target application and field rejects due to the additional costs generated and the damage to the reputation of the product in the market.

A progressive increase of the total stiffness $K_{M S}(x)$ at high positive and negative displacement as shown in Fig. 8 is desired in traditional passive transducer design to limit the peak displacement and to operate the voice coil at a safe distance to any boundaries [5]. However, a steep slope in $K_{M S}(x)$ generates high stress and fatigue in the surround for $\mathrm{x}>7 \mathrm{~mm}(\operatorname{area} \mathrm{A})$.

In active systems with reliable overload protection and voice coil stabilization, a low stiffness $K_{M S}(x)$ in a defined operation range - $X_{M A X}<x<X_{M A X}$ gives more efficiency at low frequencies $f<2 f_{s}$ and causes less fatigue in the material. However, the suspension should still provide enough radial and rotational stiffness to center the coil in gap and

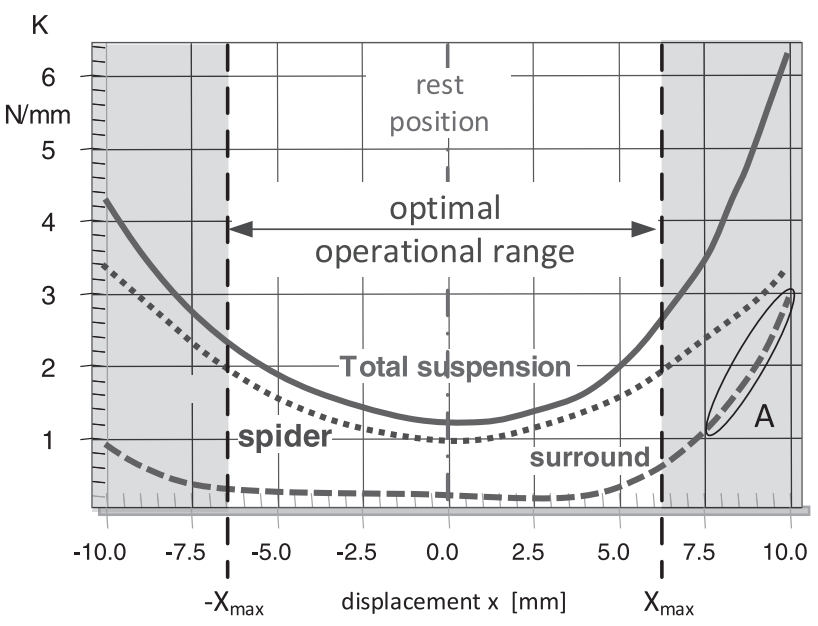

Fig. 8. Defining the optimal operational range based on the nonlinear stiffness characteristics $K_{M S}(\mathrm{x})$ of the total suspension and its components

to suppress rocking modes potentially generating voice coil rubbing.

If either a significant change of transversal stiffness $K_{M S}(x$, DUC $)$ or a high voice coil offset $X_{0}(t$, DUC $)$ is detected in a particular DUC at a later time in the final application, the adaptive, nonlinear control system reduces the operating range even further by using a lower protection limit $X_{\text {prot }}<X_{M A X}$ [25]. This Safe Mode of operation can ensure a proper suspension behavior under extreme conditions (e.g., winter) and prevents a total failure of the product by an old suspension.

\subsection{Reduced Cost}

The new software opportunities can be used to reduce the cost of the hardware components and the assembling. For example, using smaller magnets and no conductive material (e.g., shorting ring) in the motor reduces the cost while providing the same or better performance than a linear design.

\subsection{Higher Integration}

Unification of signal processing, amplification, and electroacoustical conversion provides further potential for cost saving and performance improvements of the audio device [26].

For example, Fig. 9 shows a smart transducer module that has a digital audio input supplied to a DSP performing all signal processing for the particular transducer. The alignment of DUC is combined with other linear filtering to realize the crossover and a particular transfer response required for beamforming in loudspeaker arrays. An external host processor can perform addition sound enhancement, control, and status monitoring if necessary.

The transducer can be perfectly matched with the amplifier saving weight, size and cost. The amplifier output stage is connected via short flexible wires directly to the voice coil using a low-cost output filter. The geometrical design 


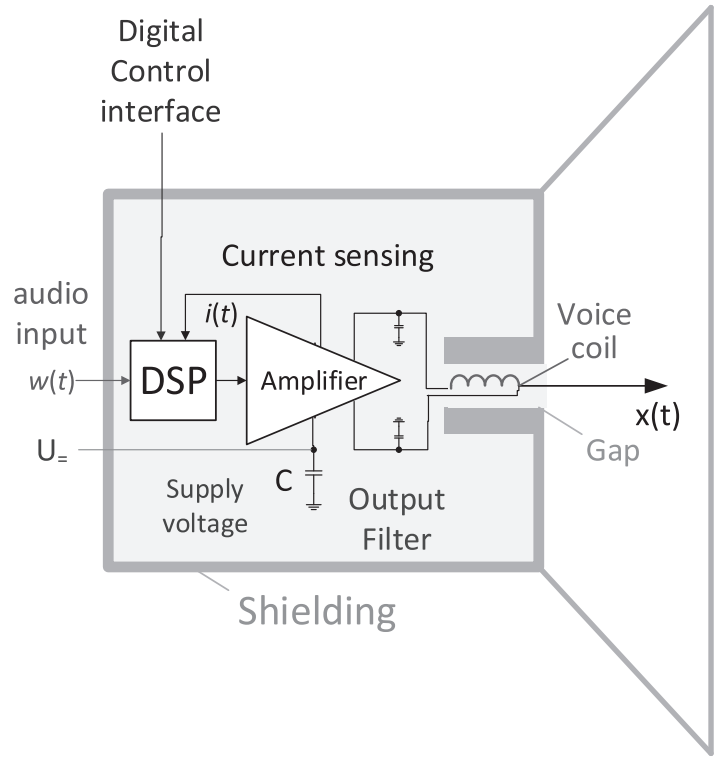

Fig. 9. Smart transducer module comprising digital signal processing (DSP), amplification, and electroacoustical conversion.

of the magnetic system (magnet, iron parts) can provide sufficient shielding to fulfill electromagnetic compatibility (EMC). Those arrangements reduce the amplifier output impedance and losses in the speaker cables, which are requirements for operating transducers with a low voice coil DC resistance (e.g $R_{E}<1 \mathrm{Ohm}$ ) giving sufficient voltage sensitivity in woofers having a high motor efficiency factor $B l^{2} / R_{E}$. This is also interesting for reducing the rail voltage in integrated circuit amplifiers to apply a less expensive silicon process. The higher peak current generated by the reduced load can be realized by a parallel connection of multiple FET transistors.

Common program material (e.g., music) has a high crest factor generating high peak values of voltage and current at the transducer terminals for a short time. Modern amplifiers reduce idling losses and increase their efficiency by reducing the rail voltage at the output stage if the output voltage is low.

The management of the rail voltage and the thermal overload protection of the amplifier can be combined with the mechanical transducer protection system in order to bring the latency in the transferred audio signal to a minimum.

Ensuring maximum efficiency of the transducer and Class D amplifier makes it possible to use the iron path or, better, the metal frame of the transducer as a heat sink for transferring the dissipated energy outwards. Since most of the power is dissipated in the voice coil, a voice coil former and diaphragm made out of metal or composite material would also improve the heat transfer.

A voltage boost converter receives an unregulated supply voltage via a cost-effective cable with a small wire diameter to supply just sufficient mean power to the amplifier. A capacitor $\mathrm{C}$ buffers the power supply in applications (e.g., battery operated) where the peak current is limited.
The effective radiation area $S_{D}$, the maximum voice coil displacement $X_{\max }$, and the maximum sound pressure level $S P L_{\max }$ as defined by IEC standard 60268-21 are the most important characteristics for selecting an optimal transducer module for a particular system design. The frequency $f_{s}$, quality factors $Q_{\mathrm{TS}}, Q_{\mathrm{MS}}$, and other linear, nonlinear, and thermal transducer parameters play a minor role in future system design.

\section{CONCLUSIONS}

Adaptive, nonlinear control can cope with time-variant properties and signal distortion limiting the maximum output of an electroacoustical transducer. This control system performs a permanent measurement of the transducer properties and state variables and generates valuable diagnostic information for quality control in manufacturing and for ensuring reliability, endurance, and robustness over product life. The potential of adaptive, nonlinear control was illustrated in this paper by using the mirror filter and other components provided by the KCS technology [27]. The performance of KCS and similar adaptive, nonlinear control technologies (find a systematic comparison in [3]) depends on the accuracy of the physical modeling of the electroacoustical system, considering only dominant nonlinearities found in the transducer reduce the DSP requirements and cost while providing sufficient performance. However, the nonlinearities in the modal vibration at higher frequencies limit the active distortion reduction at higher frequencies (e.g., intermodulation at $550 \mathrm{~Hz}$ and above $1 \mathrm{kHz}$ in Fig. 4). At the moment, this problem can only be solved by changing the geometry of the surround, cone, and other soft parts, but it is very likely that future research on nonlinear modal vibration also provides a software solution for this problem. Software algorithms and hardware components complement each other and change the paradigm in loudspeaker and headphone design.

The optimal use of all resources such as material, energy, and manufacturing effort become more important than a desired amplitude response and low distortion generated in a passive system without DSP. Systematic design methods presented for the loudspeaker system [1] and the electrodynamical transducer [2] exploit nonlinearities and modal vibrations intentionally for generating more output in smaller loudspeaker systems and reducing power consumption in portable applications with limited battery capacity. The efficiency of the electroacoustical conversion becomes the key property for speaker optimization.

Only the combination of software and hardware can improve the reliability over product life. Adaptive control operates the in the intended working range where hardware design provides sufficient robustness.

There are different ways for integrating adaptive, nonlinear control software in available host DSP or in smart amplifiers with current sensing. The additional integration cost in modern integrated circuits can easily compete with the savings in magnet cost. This opens the way to "smart" transducer modules which merge DSP, amplification, and transduction that simplify the loudspeaker system design 
and provide high performance for multichannel processing in 3D sound applications.

\section{REFERENCES}

[1] W. Klippel, "Green Speaker Design (Part 1: Optimal Use of System Resources)," presented at the 146th Convention of the Audio Engineering Society (2019 Mar.), convention paper 10138.

[2] W. Klippel, "Green Speaker Design (Part 2: Optimal Use of Transducer Resources)," presented at the 146th Convention of the Audio Engineering Society (2019 Mar.), convention paper 10139.

[3] W. Klippel, "Active Compensation of Transducer Nonlinearities," presented at the 23rd Int. Conference: Signal Processing in Audio Recording and Reproduction (2003 May), paper \#2.

[4] W. Klippel, "The Mirror Filter - A New Basis for Reducing Nonlinear Distortion Reduction and Equalizing Response in Woofer Systems", J. Audio Eng. Soc., vol. 32, no. 9, pp. 675-691, (1992 Sep.).

[5] W. Klippel, "Loudspeaker Nonlinearities - Causes Parameters, Symptoms," J. Audio Eng. Soc., vol. 54, no. 10, pp 907-939 (2006 Oct.).

[6] E. Hänsler and G. Schmidt, "Chapter 7: Nonlinear Acoustic Echo Cancellation," in Topics in Acoustic Echo and Noise Control, pp. 205-261 (Springer, Berlin, Germany, 2006).

[7] W. Klippel, "Adaptive Stabilization of Electrodynamic Transducers," J. Audio Eng. Soc., vol. 63, no. 3, pp. 154-160 (2015 Mar.).

[8] W. Klippel, "Mechanical Overload Protection of Loudspeaker Systems," J. Audio Eng. Soc., vol. 64, no. 10, pp. 771-783 (2016 Oct.).

[9] K. M. Pedersen, "Thermal Overload Protection of High-Frequency Loudspeakers," Rep. of final year dissertation, University of Salford (2002).

[10] W. Klippel, "Nonlinear Modeling of the Heat Transfer in Loudspeakers," J. Audio Eng. Soc., vol. 52, no. 1/2, pp. 3-25, (2004 Feb.).

[11] W. Klippel, "Nonlinear Adaptive Controller for Loudspeakers with Current Sensor," presented at the 106th Convention of the Audio Engineering Society (1999 May), convention paper 4864.

[12] L. Beranek and T. Mellow, Acoustics: Sound Field and Transducers (Academic Press, Amsterdam, the Netherlands, 2012).

[13] G. Ramos and J. Lopez, "Filter Design Method for Loudspeaker Equalization Based on IIR Parametric Fil- ters," J. Audio Eng. Soc., vol. 54, no. 12 pp. 1162-1178 (2006 Dec.).

[14] W. Klippel, "Adaptive Nonlinear Control of Loudspeaker Systems," J. Audio Eng. Soc., vol. 46, no. 11, pp. 939-954 (1998 Nov.).

[15] W. Klippel, "Mechanical Fatigue and Load-Induced Aging of Loudspeaker Suspension," presented at the 131st Convention of Audio Engineering Society (2011 Oct.), convention paper 8474.

[16] D. B. Keele, "Maximum Efficiency of Direct Radiator Loudspeakers," presented at the 91 st Convention of the Audio Engineering Society (1991 Oct.), convention paper 3193.

[17] W. M. Leach, Introduction to Electroacoustics and Audio Amplifier Design (Kendall/Hunt Dubuque, Iowa, 2003).

[18] N. E. Iversen, A. Knott, M. A. E. Anderson, "Relationship Between Voice Coil Fill Factor and Loudspeaker Efficiency," J. Audio Eng. Soc., vol. 64, no. 4, pp. 241-252 (2016 Apr.).

[19] Sound System Equipment - Part 5: Loudspeakers, IEC 60268-5:2003+AMD1:2007.

[20] Sound System Equipment - Electro-acoustical Transducers - Measurement of Large Signal Parameters, IEC, 62458:2010.

[21] Sound System Equipment - Part 21: Acoustical (output based) Measurements, IEC, 60268-21:2018.

[22] D. A. Anderson, Driver-Array Based Flat-Panel Loudspeakers: Theoretical Background and Design Guidelines, Ph.D. thesis, University of Rochester (2017).

[23] P. J. Hegarty, K. F. Jørgensen, J. J. Larsen, X. Ma "Nonlinear Distortion Reduction in Sound Zones by Constraining Individual Loudspeaker Control Effort," $J$. Audio Eng. Soc., vol. 57, No. 9, pp. 641-654 (2019 Sep.).

[24] W. Klippel, "Minimizing Costs in Audio Devices through Efficient End-of-Line," presented at the 145th Convention of Audio Engineering Society (2018 Oct.), convention paper 10049.

[25] S. Irrgang and W. Klippel, "Audio System Evaluation with Music Signals," presented at the 2017 AES International Conference on Automotive Audio (2017 Aug.), conference paper P4-2.

[26] J. Boehm, "A Distributed Audio System for Automotive Applications," presented at the 144th Convention of the Audio Engineering Society (2004 May), eBrief 450.

[27] KLIPPEL Controlled Sound (KCS), https:// www.klippel.de/products/klippel-controlled-sound.html 


\section{THE AUTHOR}

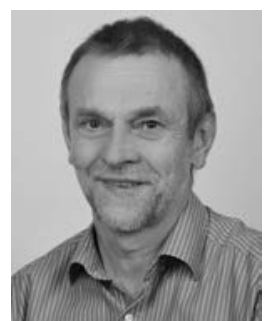

Wolfgang Klippel

Wolfgang Klippel studied electrical engineering at the University of Technology in Dresden, in the former East Germany, where his initial studies focused on speech recognition. Afterwards, he joined a loudspeaker company in the eastern part of Germany, where he was engaged in transducer modeling, acoustic measurement, and psychoacoustics. He later returned to his studies and received a Ph.D. in Technical Acoustics in 1987.

After spending a postdoctoral year at the Audio Research Group in Waterloo, Canada, and working at Harman/JBL in
Northridge, California, he returned to Dresden in 1997 and founded Klippel GmbH, a company that develops novel control and measurement systems dedicated to loudspeakers and other transducers.

Dr. Klippel has also been engaged as Professor of Electroacoustics at the University of Technology in Dresden since 2007. His papers and tutorials on loudspeaker modeling and measurement — particularly those on large signal behavior and physical distortion mechanisms - are considered reference works in the field. 